\title{
Solving Systems of Linear Inequalities by Bound Propagation
}

\author{
Konstantin Korovin^ and Andrei Voronkov ${ }^{\star \star}$ \\ The University of Manchester
}

\begin{abstract}
In this paper we introduce a new method for solving systems of linear inequalities. The algorithm incorporates many state-of-the-art techniques from DPLL-style reasoning. We prove soundness, completeness and termination of the method.
\end{abstract}

\section{Introduction}

There are several well-known methods for linear programming and solving systems of linear inequalities over the rational or real numbers. These are the Fourier-Motzkin variable elimination method, simplex, the interior point method (see, [7] for an overview), a recent conflict resolution method [2] and the GDPLL method [5]. In this paper we introduce a new method. Interestingly, this method is rather different from the previously known methods in that it incorporates ideas recently developed in the SAT solving community: namely, DPLL [1], unit propagation, dynamic variable ordering, lemma learning and backjumping [4], see also [6] for the state-of-the-art exposition of DPLL related techniques.

The method works by assigning values to variables and using the assigned values to derive bounds on other variables, using bound propagation. The process of assigning values either terminates with a solution, or results in inconsistent bounds derived by bound propagation. In the latter case we learn a new inequality, which we call a collapsing inequality, which is also used to derive a bound on a variable excluding a previously done assignment. After that we either obtain inconsistent bounds, which means that the system is unsatisfiable or change the assignment to conform to the new bound. The algorithm incorporates many ideas developed in SAT solving, such as clause learning, backjumping and dynamic variable ordering. Another interesting property of the algorithm is that the number of inequalities at each stage can be kept linear in the number of variables and the number of input inequalities.

For those familiar with DPLL, the informal description of the method above may look familiar. However, there are fundamental differences between the two methods. Firstly, it turns out that bound propagation can be non-terminating, so we have to impose some restrictions on it. Secondly, unlike propositional DPLL, there exists an infinite possible number of bounds and values for a variable, so making an algorithm that terminates is highly non-trivial.

\footnotetext{
* Supported by a Royal Society University Research Fellowship

${ }^{\star \star}$ Partially supported by an EPSRC grant
} 
The rest of this paper is organised as follows. Section 2 introduces definitions related to systems of linear inequalities. We define notions of bound (on a variable), context as a set of bounds, and inference rules of resolution and bound-resulting resolution on linear inequalities. We also introduce bound propagation as a sequence of bound-resulting resolution inferences. Section 3 introduces a fundamental notion of collapsing inequality. We give an algorithm for extracting collapsing inequalities from resolution proofs. We show that, in the case of bound propagation, the extracted collapsing inequality can be used to collapse a bound propagation derivation into a single inference by boundresulting resolution.

In Section 4 we introduce our algorithm for solving systems of linear inequalities using bound propagation and other rules. Section 5 gives an example of how this algorithm works. In Section 6 we show soundness, completeness and termination of the algorithm. Proofs that did not fit can be found in the full version of this paper [3].

\section{Preliminaries}

We will denote variables by $x$, rational constants by $c$ and positive rational constants by $d$, maybe with indices. We call a literal a variable $x$ or its negation $-x$ and denote literals by $l$. Literals of the forms $x$ and $-x$ are said to be complementary. A literal complementary to a literal $l$ will be denoted by $\bar{l}$. Note that every linear inequality can be written in the form

$$
d_{1} l_{1}+\cdots+d_{n} l_{n}+c \geq 0 .
$$

where the variables of the $l_{i}$ 's are pairwise different. Note that all the constants $d_{i}$ 's are positive by our choice of notation. We say that inequality (1) contains literals $l_{1}, \ldots, l_{n}$. An inequality is called trivial if it contains no variables. It is straightforward to adapt all our considerations to systems also containing strict inequalities and equalities, which for simplicity we do not consider in this paper.

We define an assignment $\sigma$ over a set of variables $X$ as a mapping from $X$ to the set of real numbers $\mathbb{R}$, i.e. $\sigma: X \rightarrow \mathbb{R}$.

For a linear term $q$ over $X$, denote by $q \sigma$ the value of $q$ after replacing all variables $x \in X$ by the corresponding values $\sigma(x)$. An assignment $\sigma$ is called a solution of a linear inequality $q \geq 0$ if $q \sigma \geq 0$ is true; it is a solution of a system of linear inequalities if it is a solution of every inequality in the system. If $\sigma$ is a solution of a linear inequality I (or a system $\mathcal{L}$ of such inequalities), we also say that $\sigma$ satisfies I (respectively, $\mathcal{L}$ ), denoted by $\sigma \models \mathrm{I}$ (respectively, $\sigma \models \mathcal{L}$ ), otherwise we say that $\sigma$ violates I (respectively, $\mathcal{L})$. A system of linear inequalities is said to be satisfiable if it has a solution.

We will denote inequalities as I, J, G, possibly with indexes and the corresponding linear terms as $I, J, G$ respectively, so $\mathrm{I}=(I \geq 0), \mathrm{J}=(J \geq 0)$ and so on. Two linear inequalities are equivalent if one can be obtained from another by multiplying by a positive constant. When we deal with linear inequalities, we will not distinguish equivalent linear inequalities. That is, we assume that the order of linear terms $d_{i} l_{i}$ in a linear expression is irrelevant and that we can multiply the inequality by any positive rational. We also assume that a trivial inequality is either $-1 \geq 0$ or $0 \geq 0$.

For an inequality I, let $\operatorname{var}(\mathrm{I})$ denote the set of all variables with non-zero coefficients in I. Similarly, for a system of inequalities $\mathcal{L}$, let $\operatorname{var}(\mathcal{L})$ denote the set 


\begin{tabular}{|c|c|}
\hline SAT & this paper \\
\hline variable & variable \\
\hline literal & literal \\
\hline clause & linear inequality \\
\hline unit clause & bound \\
\hline resolution & resolution \\
\hline unit-resulting resolution & bound-resulting resolution \\
\hline unit propagation & bound propagation \\
\hline
\end{tabular}

Table 1. Correspondence between the SAT terminology and our terminology

$\cup_{\mathrm{I} \in \mathcal{L}} \operatorname{var}(\mathrm{I})$. We say that a system of inequalities $\mathcal{L}$ implies an inequality $\mathrm{I}$, if every solution to $\mathcal{L}$ is also a solution to $\mathrm{I}$. We say that an inequality $\mathrm{I}$ is a non-negative linear combination of inequalities $I_{1}, \ldots, I_{k}$, if $I$ is of the form $\alpha_{1} I_{1}+\cdots+\alpha_{k} I_{k} \geq 0$ where $\alpha_{i} \geq 0$ for $1 \leq i \leq k$, in this case we also write $\mathrm{I}=\alpha_{1} \mathrm{I}_{1}+\cdots+\alpha_{k} \mathrm{I}_{k}$. It is easy to see that any non-negative linear combination of inequalities from $\mathcal{L}$ is implied by $\mathcal{L}$. An inequality $\mathrm{I}$ of the form $d_{1} l_{1}+\cdots+d_{n} l_{n}+c \geq 0$ improves an inequality $\mathrm{I}^{\prime}$ if either I is $-1 \geq 0$ or $\mathrm{I}^{\prime}$ is of the form $d_{1} l_{1}+\cdots+d_{n} l_{n}+c^{\prime} \geq 0$ and $c^{\prime} \geq c$.

LEMMA 2.1 If an inequality I improves $I^{\prime}$ then $I$ implies $I^{\prime}$.

Proof Let I be of the form $d_{1} l_{1}+\cdots+d_{n} l_{n}+c \geq 0$. If I is $-1 \geq 0$ then the lemma trivially holds. Assume $\mathrm{I}$ is not $-1 \geq 0$ and $\mathrm{I}^{\prime}$ is of the form $d_{1} l_{1}+\cdots+d_{n} l_{n}+c^{\prime} \geq 0$, where $c^{\prime} \geq c$. Then $\mathrm{I}^{\prime}=\mathrm{I}+\left(c^{\prime}-c \geq 0\right)$, hence $\mathrm{I}$ implies $\mathrm{I}^{\prime}$.

DEFINITION 2.2 (BOUNDS) A bound on a literal $l$ is an inequality of the form $l+c \geq$ 0 . A pair of bounds $l+c_{1} \geq 0$ and $\bar{l}+c_{2} \geq 0$ on two complementary literals is contradictory if $c_{1}+c_{2}<0$, in this case we will also say that $l+c_{1} \geq 0$ contradicts $\bar{l}+$ $c_{2} \geq 0$. A bound is either a bound on a literal, or a trivial inequality. Trivial inequalities will also be called trivial bounds. A bound $l+c_{1} \geq 0$ is said to strictly improve a bound $l+c_{2} \geq 0$ if $c_{2}>c_{1}$.

DEFinition 2.3 (CONTEXT) Let $B$ be a finite set of non-trivial bounds. $B$ is called a context if it contains no contradictory pair of bounds. A bound $\mathrm{b}$ is called redundant in $B$ if some bound in $B$ strictly improves $\mathrm{b}$. We say that a bound $\mathrm{b}$ contradicts to a context $B$ if some bound in $B$ contradicts $\mathrm{b}$.

By our definition contexts are always satisfiable. It is easy to see that a bound $\mathrm{b}$ is implied by a context $B$ if and only if either $\mathrm{b} \in B$ or $\mathrm{b}$ is redundant in $B$.

Our aim now is to introduce an inference system on linear inequalities. This inference system will have inference rules similar to those used in the resolution calculus. To emphasise the analogy to the resolution calculus we will use terminology similar to the one used in the theory of resolution.

For the readers familiar with the resolution calculus for propositional logic and the DPLL method we define a correspondence between the notions introduced in this paper and those used in propositional satisfiability in Table 1. 
DEFINITION 2.4 (Resolution) Let $\mathrm{I}_{1}, \mathrm{I}_{2}$ be linear inequalities such that $\mathrm{I}_{1}$ has the form $d_{1} x+I_{1}^{\prime} \geq 0$ and $\mathrm{I}_{2}$ has the form $-d_{2} x+I_{2}^{\prime} \geq 0$ for some variable $x$. We say that the linear inequality

$$
d_{2} I_{1}^{\prime}+d_{1} I_{2}^{\prime} \geq 0
$$

is a resolvent of $\mathrm{I}_{1}$ and $\mathrm{I}_{2}$ upon $x$. We consider resolvent as a symmetric relation, that is, a resolvent of $\mathrm{I}_{1}$ and $\mathrm{I}_{2}$ upon $x$ is also a resolvent of $\mathrm{I}_{2}$ and $\mathrm{I}_{1}$ upon $x$. Resolution is the inference rule deriving a resolvent from two linear inequalities.

For example, consider two clauses $x_{1}+2 x_{2}+x_{3}+3 \geq 0$ and $-2 x_{1}-3 x_{2}+5 \geq 0$. Then their resolvent upon $x_{1}$ is $x_{2}+2 x_{3}+11 \geq 0$ and their resolvent upon $x_{2}$ is $-x_{1}+3 x_{3}+19 \geq 0$. Note that any resolvent of two inequalities is a consequence of these inequalities. Also note that resolution is compatible with equivalence on linear inequalities. That is if we replace inequalities in the premise of a resolution inference by equivalent inequalities then the conclusion of the new inference will be equivalent to the conclusion of the original inference.

Any application of resolution to a bound $\mathrm{b}$ and a linear inequality I eliminates a variable from $I$ in the following sense: the variables of the resolvent are the variables of I minus the variable of $b$. Thus, if we repeatedly apply resolution to a linear inequality of $n$ variables $x_{1}, \ldots, x_{n}$ and bounds on variables $x_{2}, \ldots, x_{n}$, all variables except $x_{1}$ in I will be eliminated and we will obtain a bound on $x_{1}$. We will formalise such repeated applications of resolution to a linear inequality and a sequence of bounds in the following definition.

DEFINITION 2.5 (Bound-Resulting Resolution) Consider any linear inequality I of the form (1). Let $\mathrm{b}_{i}$ be bounds of the form $\bar{l}_{i}+c_{i} \geq 0$, where $i=2, \ldots, n$, on literals complimentary to literals in $I$. Then one can derive (by a sequence of resolution inferences) from $\mathrm{b}_{2}, \ldots, \mathrm{b}_{n}$ and I the following bound $\mathrm{b}$ on $l_{1}$ :

$$
l_{1}+\left(c+d_{2} c_{2}+\ldots+d_{n} c_{n}\right) / d_{1} \geq 0 .
$$

We will say that $\mathrm{b}$ is obtained by bound-resulting resolution from $\mathrm{b}_{2}, \ldots, \mathrm{b}_{n}$ and $\mathrm{I}$.

Likewise, let $\mathrm{b}_{i}$ be bounds of the form $\bar{l}_{i}+c_{i} \geq 0$, where $i=1, \ldots, n$, on literals complimentary to literals in $I$. Then one can derive (by a sequence of resolution inferences) from $\mathrm{b}_{1}, \ldots, \mathrm{b}_{n}$ and $\mathrm{I}$ the following trivial inequality:

$$
c+d_{1} c_{1}+d_{2} c_{2}+\ldots+d_{n} c_{n} \geq 0 .
$$

In this case we will also say that this trivial inequality is obtained by bound-resulting resolution from $\mathrm{b}_{1}, \ldots, \mathrm{b}_{n}$ and $\mathrm{I}$.

We can consider resolution and bound-resulting resolution as inference rules and put together sequences of resolution steps to form a derivation, that is, a tree consisting of inferences. For example, the following

$$
\frac{\frac{x_{4}-1 \geq 0 \quad x_{3}-x_{4}+1 \geq 0}{x_{3} \geq 0} \quad-x_{2} \geq 0 \quad x_{4}-1 \geq 0 \quad x_{1}+x_{2}-x_{3}-x_{4} \geq 0}{x_{1}-1 \geq 0}
$$


is a derivation of the bound $x_{1}-1 \geq 0$ from two bounds $x_{4}-1 \geq 0$ and $-x_{2} \geq 0$ and two inequalities $x_{3}-x_{4}+1 \geq 0$ and $x_{1}+x_{2}-x_{3}-x_{4} \geq 0$. This derivation uses two bound propagation inferences.

By repeated applications of bound-resulting resolution we can repeatedly derive new bounds. Such repeated applications are formalised in the following definition.

Definition 2.6 (Bound Propagation) Let $B$ be a context and $\mathcal{L}$ a system of linear inequalities. A bound propagation from $B$ and $\mathcal{L}$ is a sequence of bounds $\mathrm{b}_{1}, \ldots, \mathrm{b}_{n}$, such that

1. $n>0$.

2. For all $k$ such that $1 \leq k \leq n$, the bound $\mathrm{b}_{k}$ is not implied by $B \cup\left\{\mathrm{b}_{1}, \ldots, \mathrm{b}_{k-1}\right\}$.

3. For all $k$ such that $1 \leq k \leq n$, the bound $\mathrm{b}_{k}$ is obtained by bound-resulting resolution from $B \cup\left\{\mathrm{b}_{1}, \ldots, \mathrm{b}_{k-1}\right\}$ and an inequality in $\mathcal{L}$.

We will also use this definition in the case when $\mathrm{b}_{n}$ is a trivial inequality.

By collecting all inferences in a tree one can regard bound propagation as a derivation of the bound (or a trivial inequality) $\mathrm{b}_{n}$ from $B$ and $\mathcal{L}$.

\section{Collapsing Inequalities}

In the DPLL procedure unit propagation always terminates since there is only a finite number of literals that can be derived. In the case of linear equalities the number of bounds is infinite, which may result in bound propagation of unrestricted length, deriving better and better bounds. This is illustrated by the following example.

EXAMPLE 3.1 Consider the context $\left\{x_{1} \geq 0\right\}$ and the following two linear inequalities

$$
\begin{aligned}
x_{2}-x_{1} & \geq 0 \\
x_{1}-x_{2}-1 & \geq 0
\end{aligned}
$$

Using $x_{1} \geq 0$ and (3) one can derive a new bound $x_{2} \geq 0$, from which, using (4) one can derive an improved bound $x_{1}-1 \geq 0$ on $x_{1}$. In a similar way from $x_{1}-1 \geq 0$ one can derive in two steps $x_{1}-2 \geq 0$, then $x_{1}-3 \geq 0$ etc.

In this section we will analyse bound propagation. First, we will show that any derivation consisting of bound propagation steps using a collection of inequalities can be collapsed into a single bound-resulting resolution inference by adding a new inequality, called a collapsing inequality.

To explain the idea of collapsing inequalities consider derivation (2). It uses two inferences to derive the bound $x_{1}-1 \geq 0$ from the context $B=\left\{x_{4}-1 \geq 0,-x_{2} \geq 0\right\}$. It also derives the bound $x_{3} \geq 0$ on the variable $x_{3}$. If we resolve the inequalities $x_{1}+x_{2}-x_{3}-x_{4} \geq 0$ and $x_{3}-x_{4}+1 \geq 0$ used in the derivation upon the variable $x_{3}$ we obtain a new inequality $x_{1}+x_{2}-2 x_{4}+1 \geq 0$. This inequality has the following interesting property: we can obtain the bound $x_{1}-1 \geq 0$ from the context $B$ using a single inference 


$$
\frac{x_{4}-1 \geq 0 \quad-x_{2} \geq 0 \quad x_{1}+x_{2}-2 x_{4}+1 \geq 0}{x_{1}-1 \geq 0} .
$$

Thus, the new inequality $x_{1}+x_{2}-2 x_{4}+1 \geq 0$ makes derivation (2) collapse into a single inference.

Let us prove a general result on collapsing inequalities and then show how to extract collapsing inequalities from resolution and bound-resulting resolution proofs. We will use Farkas's Theorem stated in the following form.

THEOREM 3.2 (Farkas) Let $\mathcal{L}$ be a system of linear inequalities. If $\mathcal{L}$ implies a linear inequality $I$ then there is a linear non-negative combination of inequalities from $\mathcal{L}$ improving $I$.

For a proof we refer to [7].

THEOREM 3.3 (Collapsing Inequalities) Let $\mathcal{L}_{1}$ and $\mathcal{L}_{2}$ be two systems of linear inequalities such that $\mathcal{L}_{1} \cup \mathcal{L}_{2}$ implies a linear inequality $I$. Then there exist two linear inequalities $I_{1}$ and $I_{2}$ such that

1. $\mathcal{L}_{1}$ implies $I_{1}$ and $\mathcal{L}_{2}$ implies $I_{2}$;

2. the system $\left\{I_{1}, I_{2}\right\}$ implies $I$.

Proof By Theorem 3.2, if $\mathcal{L}_{1} \cup \mathcal{L}_{2}$ implies a linear inequality I then there is a nonnegative linear combination of inequalities from $\mathcal{L}_{1} \cup \mathcal{L}_{2}$ which implies I. This combination can be represented in the form

$$
\alpha_{1} \mathrm{~J}_{1}+\cdots+\alpha_{k} \mathrm{~J}_{k}+\beta_{1} \mathrm{G}_{1}+\cdots+\beta_{m} \mathrm{G}_{m},
$$

where $\mathrm{J}_{i} \in \mathcal{L}_{1}, \alpha_{i} \geq 0$ for $1 \leq i \leq k$ and $\mathrm{G}_{i} \in \mathcal{L}_{2}, \beta_{i} \geq 0$ for $1 \leq i \leq m$.

We define $\mathrm{I}_{1}=\alpha_{1} \mathrm{~J}_{1}+\cdots+\alpha_{k} \mathrm{~J}_{k}$ and $\mathrm{I}_{2}=\beta_{1} \mathrm{G}_{1}+\cdots+\beta_{m} \mathrm{G}_{m}$. It is straightforward to check that $I_{1}$ and $I_{2}$ satisfy conditions 1-2 of the theorem.

We will call inequalities $I_{1}$ and $I_{2}$ satisfying the conditions of Theorem 3.3 collapsing for I w.r.t. $\mathcal{L}_{1}$ and $\mathcal{L}_{2}$ respectively.

Let us show how to effectively extract collapsing inequalities from resolution proofs.

THEOREM 3.4 Let $\mathcal{L}_{1}$ and $\mathcal{L}_{2}$ be two systems of linear inequalities and $\Pi$ be a resolution (or a bound-resulting resolution) derivation of an inequality I from inequalities in $\mathcal{L}_{1} \cup \mathcal{L}_{2}$. Then inequalities $\mathrm{I}_{1}$ and $\mathrm{I}_{2}$, collapsing for I w.r.t. $\mathcal{L}_{1}$ and $\mathcal{L}_{2}$ respectively, can be constructed in time polynomial in the size of $\Pi$.

Proof Since any bound-resulting resolution derivation can be considered as a special case of a resolution derivation, we will only prove this theorem for resolution derivations.

The proof is by induction on the depth of $\Pi$. We will prove a slightly stronger, yet equivalent statement: our construction will also imply $I=I_{1}+I_{2}$.

Base case. Suppose $\Pi$ has I as the (only) leaf, then $I \in \mathcal{L}_{1} \cup \mathcal{L}_{2}$. If I $\in \mathcal{L}_{1}$ then we define $I_{1} \stackrel{\text { def }}{=} I$ and $I_{2} \stackrel{\text { def }}{=}(0 \geq 0)$. The case $I \in \mathcal{L}_{2}$ is similar. 
Inductive case. Let I be a conclusion of an inference in $\Pi$ with premises $\mathrm{J}$ and G:

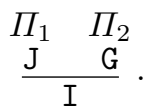

By the induction hypothesis, we can construct pairs of collapsing inequalities $\mathrm{J}_{1}, \mathrm{~J}_{2}$ for $\Pi_{1}$ and $G_{1}, G_{2}$ for $\Pi_{2}$. Since $I$ is obtained by resolution from $J$ and $G$, we have that $\mathrm{I}=\alpha \mathrm{J}+\beta \mathrm{G}$ for some coefficients $\alpha>0$ and $\beta>0$. Define $\mathrm{I}_{1} \stackrel{\text { def }}{=} \alpha \mathrm{J}_{1}+\beta \mathrm{G}_{1}$ and $\mathrm{I}_{2} \stackrel{\text { def }}{=} \alpha \mathrm{J}_{2}+\beta \mathrm{G}_{2}$. By the induction hypothesis, $\mathcal{L}_{1}$ implies $\left\{\mathrm{J}_{1}, \mathrm{G}_{1}\right\}$, hence $\mathcal{L}_{1}$ implies $I_{1}$. Likewise, we have $\mathcal{L}_{2}$ implies $I_{2}$. By the induction hypothesis we also have $\mathrm{J}=\mathrm{J}_{1}+\mathrm{J}_{2}$ and $\mathrm{G}=\mathrm{G}_{1}+\mathrm{G}_{2}$. It remains to prove $\mathrm{I}_{1}+\mathrm{I}_{2}=\mathrm{I}$. To this end, note that $\mathrm{I}_{1}+\mathrm{I}_{2}=\left(\alpha \mathrm{J}_{1}+\beta \mathrm{G}_{1}\right)+\left(\alpha \mathrm{J}_{2}+\beta \mathrm{G}_{2}\right)=\alpha\left(\mathrm{J}_{1}+\mathrm{J}_{2}\right)+\beta\left(\mathrm{G}_{1}+\mathrm{G}_{2}\right)=\alpha \mathrm{J}+\beta \mathrm{G}=\mathrm{I}$.

Let us note that with additional bookkeeping we can obtain an explicit representation for the collapsing inequalities $I_{1}$ and $I_{2}$ as non-negative combinations of inequalities from $\mathcal{L}_{1}\left(\mathcal{L}_{2}\right.$ respectively).

Let us now consider bound-resulting resolution inferences between a context $B$ and a set of inequalities $\mathcal{L}$.

THEOREM 3.5 Let b be a (possibly trivial) bound derived from a context $B$ and a set $\mathcal{L}$ of linear inequalities by bound propagation. Then there exists a linear inequality I such that

1. $\mathcal{L}$ implies $I$, and

2. either (i) $I$ is $-1 \geq 0$, or (ii) there is a bound $b^{\prime}$ improving $b$ which can be derived from $B$ and I by a single bound-resulting resolution inference.

Moreover, the bound $b^{\prime}$ and inequality I can be constructed in time polynomial in the size of the derivation by bound propagation.

The proof is given in Appendix A. Let us only note that we can take I to be a collapsing inequality for b w.r.t. $\mathcal{L}$, which by Theorem 3.4 , can be computed in polynomial time from the bound-resulting resolution proof of $\mathrm{b}$.

\section{Bound Propagation Algorithm}

In this section we introduce the bound propagation algorithm for solving systems of linear inequalities, called BPA. BPA will be presented using a system of derivation (transition) rules which are applied to states of BPA. A state is a triple $(S, \mathcal{L}, \epsilon)$ where $S$ is a sequence of annotated bounds, called bound stack, $\mathcal{L}$ is a system of inequalities and $\epsilon$ is either the empty set or a set consisting of one bound, called conflicting bound. We denote a state $(S, \mathcal{L}, \epsilon)$ as $S \| \mathcal{L}, \epsilon$ and in the case when $\epsilon=\emptyset$ as $S \| \mathcal{L}$. The bounds in $S$ are annotated with information reflecting on how the bounds were introduced. Each bound b in $S$ is either:

- a decision bound, denoted $\mathrm{b}^{d}$, or

- a propagation bound, denoted as $\mathrm{b}^{p}$. 
We say that a bound $\mathrm{b}$ in $S$ is below a bound $\mathrm{b}^{\prime}$ in $S$ if b occurs before $\mathrm{b}^{\prime}$ (in the order of their occurrences in $S$ ), in this case $\mathrm{b}^{\prime}$ is also called above b. In other words, we consider the stack as growing upwards.

Every propagation bound in the stack will be obtained by bound propagation from bounds below it and linear inequalities in $\mathcal{L}$. Consider a bound stack $S$. Let b be a propagation bound in the stack. We refer to the corresponding bound-resulting resolution derivation of $\mathrm{b}$ from bounds below it as $\Pi_{\mathrm{b}}$. Based on Theorems (3.4-3.5) we can calculate a collapsing inequality from $\Pi_{\mathrm{b}}$, which we call the collapsing inequality for $b$ under $S$ and denote by $\mathrm{CI}_{\mathrm{b}}$, such that (i) $\mathrm{CI}_{\mathrm{b}}$ is implied by $\mathcal{L}$ and (ii) a bound improving $\mathrm{b}$ can be derived by a single bound-resulting resolution inference from $\mathrm{CI}_{\mathrm{b}}$ and decision bounds in $S$ below b.

An initial state of BPA is a state of the form $S_{0} \| \mathcal{L}_{0}$ where $S_{0}$ is the empty sequence, and $\mathcal{L}_{0}$ is the system of inequalities we want to solve. Let us state invariants on BPA states which will be preserved by BPA derivations. These invariants either trivially follow from the BPA rule definitions or will be proved later. Consider a BPA state $S \| \mathcal{L}, \epsilon$ which is obtained from an initial state by a sequence of applications of BPA derivation rules.

Invariant 1. The set of all bounds in $S$ is consistent. In other words, we can consider $S$ as a context.

We call a sequence consisting of a pair of bounds $\left\langle x-c \geq^{d} 0,-x+c \geq^{d} 0\right\rangle$ a decision pair on $x$ with the decision value $c$. To simplify the notation we will also write such a decision pair as $x^{c}$. If a stack contains a decision pair on $x$, we will call $x$ a decision variable of this stack.

Invariant 2. $S$ is of the form $U_{0} x_{1}^{c_{1}} U_{1} \ldots x_{k}^{c_{k}} U_{k}$ where $k \geq 0$ and (i) all $x_{1}^{c_{1}}, \ldots, x_{k}^{c_{k}}$ are decision pairs, (ii) each $U_{i}$ contains no decision bounds for $0 \leq i \leq k$, (iii) for any variable $x$ there is at most one decision pair on $x$ in $S$. We say that bounds in $U_{i}$ are implied bounds of the decision level $i$ for $0 \leq i \leq k$, and the decision level of $S$ is $k$.

Denote a restriction of the stack $S$ to bounds below a decision pair $x^{c}$ as $S_{<x^{c}}$ (including $x^{c}$ as $S_{\leq x^{c}}$ ), or simply $S_{<l}\left(S_{\leq l}\right.$, respectively) when we are not concerned with the decision value and $\operatorname{var}(l)=x$.

Invariant 3. Any propagation bound b in $S$ is not implied by the set of the bounds below b.

Let us note that Invariants $(2,3)$ imply that if $S$ contains a decision pair on a variable $x$ then there are no bounds on $x$ above this decision pair.

A bound $\mathrm{b}$ is called decision-derived from an inequality $\mathrm{I}$ and a stack $S$ if either (i) both $\mathrm{I}$ and $\mathrm{b}$ are $-1 \geq 0$, or (ii) $\mathrm{I}$ is of the form $l+I \geq 0$, where all variables in $\mathrm{I}$ are decision variables in $S_{\leq l}$, and b either coincides with I, or is obtained by a single bound-resulting resolution inference from I and decision bounds in $S_{<l}$.

With each decision pair $x^{c}$ in $S$ we associate a pair of sets $B C I_{x}=\left\langle U S_{x}, L S_{x}\right\rangle$ called a bounding collapsing interval on $x$ with the following properties. The set $L S_{x}$ (respectively, $U S_{x}$ ) is either empty or consists of a single inequality $L_{x}$ (respectively, $U_{x}$ ) of the form $x+I \geq 0(-x+I \geq 0$, respectively), where all variables in $I$ are decision variables in $S_{<x}$. We denote by $l b_{x}$ the bound on $x$ decision-derived from $L_{x}$ 
and $S$, called the lower collapsing bound on $x$ and similarly by $u b_{x}$ the bound on $x$ decision-derived from $U_{x}$ and $S$, called the upper collapsing bound on $x$.

Invariant 4. For any decision pair $x^{c}$ in $S$ with the associated bounding collapsing pair $B C I_{x}$, inequalities in $B C I_{x}$ are implied by $\mathcal{L}$.

For example, consider a system of inequalities $\mathcal{L}$ :

$$
\begin{array}{r}
-y+x-2 z+3 \geq 0 \\
y-x-3 z+1 \geq 0 \\
z \geq 0
\end{array}
$$

and a stack

$$
S=\left\langle z \geq^{p} 0, x^{1}, y^{2}\right\rangle
$$

Assume that with the first decision pair we associated a bounding collapsing pair consisting of empty sets, and with the second the pair $\langle\{-y+x+3 \geq 0\},\{y-x+1 \geq$ $0\}\rangle$. Invariant 4 is satisfied since both $-y+x+3 \geq 0$ and $y-x+1 \geq 0$ are implied by $\mathcal{L}$. Note that the assignment $\{y \mapsto 2\}$ satisfies the corresponding upper and lower collapsing bounds $u b_{y}=(-y+4 \geq 0)$ and $l b_{y}=(y \geq 0)$.

Invariant 5. If $\epsilon$ consists of a conflicting bound $\mathrm{b}$ then $\mathrm{b}$ contradicts to a decision bound in $S$. With each conflicting bound b we associate a conflicting collapsing inequality $\mathrm{CCI}_{\mathrm{b}}$ satisfying the following. Conflicting collapsing inequality $\mathrm{CCI}_{\mathrm{b}}$ is implied by $\mathcal{L}$, and b is the bound decision-derived from $\mathrm{CCI}_{\mathrm{b}}$ and $S$.

We define the bound-propagation depth of bounds in a bound-propagation derivation w.r.t. $S$ inductively as follows. If a bound b is a decision bound in $S$ or a bound in $\mathcal{L}$ then the bound propagation depth of $\mathrm{b}$ is $b p d(\mathrm{~b})=0$. If a bound $\mathrm{b}$ is obtained by a bound-resulting resolution inference from bounds $\mathrm{b}_{1}, \ldots, \mathrm{b}_{k}$ and an inequality in $\mathcal{L}$ then the bound-propagation depth of $\mathrm{b}$ is $\operatorname{bpd}(\mathrm{b})=\max \left\{\operatorname{bpd}\left(\mathrm{b}_{i}\right) \mid 1 \leq i \leq k\right\}+1$.

Invariant 6. We restrict bound-propagation depth of propagation bounds in $S$ by an a priory fixed constant denoted $D \geq 0$.

During backjumping (rules (LBBC) and (UBBC)) we can resolve collapsing inequalities. In order to show that the number of such resolvents is finite we need a notion of the resolution rank.

The resolution rank of inequalities is defined by induction as follows. The set of inequalities of rank 0 , denoted $R I_{0}$, consists of all inequalities in $\mathcal{L}$ together with all collapsing inequalities obtained from bound-propagation derivations of depth $\leq D+$ 1 , from $\mathcal{L}$ and a set of bounds. Assume that $R I_{k}$ is defined then $R I_{k+1}$ consists of all inequalities in $R I_{k}$ together with all inequalities obtained by a single resolution inference from inequalities in $R I_{k}$.

LEMMA 4.1 For any (finite) set of inequalities $\mathcal{L}$ and any non-negative integers $D$ and $k$, the set $R I_{k}$ is finite.

The proof is given in Appendix B.

Invariant 7. Consider the conflicting collapsing inequality $\mathrm{CCI}_{\mathrm{b}}$ associated with a conflicting bound $\mathrm{b}$ of the form $l+c \geq 0$. Denote the number of all variables in $\mathcal{L}$ as $|\operatorname{var}(\mathcal{L})|$ and the number of decision variables in $S_{\leq l}$ as $\left|\operatorname{var}_{d}\left(S_{\leq l}\right)\right|$. Then 
$\mathrm{CCI}_{\mathrm{b}} \in R I_{|\operatorname{var}(\mathcal{L})|-\left|\operatorname{var}_{d}\left(S_{\leq l}\right)\right|}$. Similarly, if $x^{c}$ is a decision variable in $S$ then $B C I_{x} \in$ $R I_{|\operatorname{var}(\mathcal{L})|-\left|\operatorname{var}_{d}\left(S_{\leq x}\right)\right| \cdot}$

We define a contradictory BPA state as a special state denoted as $\perp$. Now we define the BPA derivation rules. When defining the rules, we assume by induction, that all invariants above are satisfied on the BPA states to which the rules are applied. Initial BPA states trivially satisfy the invariants.

\section{Bound Propagation (BP):}

$$
S\left\|\mathcal{L} \Rightarrow_{B P} S \mathrm{~b}^{p}\right\| \mathcal{L}, \text { where }
$$

1. b is in $\mathcal{L}$, or obtained by bound propagation from $S$ and $\mathcal{L}$,

2. $\operatorname{bpd}(\mathrm{b}) \leq D$,

3. b is consistent with $S$,

4. b is not implied by $S$.

\section{Decide (D):}

$$
S\left\|\mathcal{L} \Rightarrow_{D} S x^{c}\right\| \mathcal{L} \text {, where }
$$

1. $x^{c}$ is a decision pair $\left\langle x-c \geq^{d} 0,-x+c \geq^{d} 0\right\rangle$, such that

2. $x$ is a variable in $\mathcal{L}$,

3. $x$ is not a decision variable in $S$,

4. bounds $x-c \geq 0$ and $-x+c \geq 0$ are consistent with $S$ and at least one bound in $x^{c}$ is not implied by $S$.

In the Decide rule above, we associate with the introduced decision pair $x^{c}$ a bounding collapsing pair $B C I_{x}=\left\langle U S_{x}, L S_{x}\right\rangle$ where $U S_{x}$ and $L S_{x}$ are defined as follows. If there is no lower bound on $x$ in $S$ then $L S_{x}$ is the empty set. Otherwise, let $x-c^{\prime} \geq 0$ be a lower bound on $x$ in $S$ which is not implied by any other bound in $S$. Note that $x-c^{\prime} \geq 0$ is a propagation bound, since by conditions on the applicability of Decide, $x$ is not a decision variable in $S$. Therefore $x-c^{\prime} \geq 0$ is derived from $\mathcal{L}$ and decision bounds in $S$ by bound propagation (of depth $\leq D$ ). We define $L S_{x}=\left\{\mathrm{CI}_{\left(x-c^{\prime} \geq 0\right)}\right\}$. The set $U S_{x}$ is defined similarly.

\section{Conflicting Bound (CB):}

$$
S\left\|\mathcal{L} \Rightarrow_{C B} S\right\| \mathcal{L},\{\mathrm{b}\}, \text { where }
$$

1. $(-1 \geq 0)$ is obtained by bound-resulting resolution from $S$ and $\mathcal{L}$,

2. $\mathrm{CI}_{(-1 \geq 0)}$ is the collapsing inequality for $(-1 \geq 0)$ under $S$, and

3. b is decision-derived from $\mathrm{CI}_{(-1 \geq 0)}$ and $S$.

In the Conflicting Bound rule above, we associate with the bound $\mathrm{b}$ the conflicting collapsing inequality $\mathrm{CCI}_{\mathrm{b}}=\mathrm{CI}_{(-1 \geq 0)}$.

\section{Contradiction $(\perp)$ :}

$$
S \| \mathcal{L},\{-1 \geq 0\} \Rightarrow_{\perp} \perp
$$


The next set of rules deals with the case when the conflicting bound $\mathrm{b}$ is a lower bound, i.e., of the form $x+c \geq 0$, these rules are (LBBV) and (LBBC). The case when the conflicting bound is of the form $-x+c \geq 0$ is similar and the corresponding rules are (UBBV) and (UBBC).

\section{Lower Bound Backjump Value (LBBV):}

$$
V x^{u} U\left\|\mathcal{L},\{x+c \geq 0\} \Rightarrow_{L B B V} V x^{v}\right\| \mathcal{L}, \text { where }
$$

1. $\operatorname{CCI}_{(x+c \geq 0)}$ is of the form $x+I \geq 0$,

2. $x+c \geq 0$ is consistent with the upper bound $u b_{x}$,

3. define $B C I_{x}^{\prime}=\left\langle U S_{x},\left\{\operatorname{CCI}_{(x+c \geq 0)}\right\}\right\rangle$,

4. $x^{v}$ is consistent with $u b_{x}^{\prime}$ and $l b_{x}^{\prime}$ corresponding to $B C I_{x}^{\prime}$.

In the (LBBV) rule above, with the new decision pair $x^{v}$ we associate the bounding collapsing pair $B C I_{x}^{\prime}$.

We use the following notation. Consider two inequalities of the form $\mathrm{I}=(x+I \geq$ $0)$ and $\mathrm{J}=(-x+J \geq 0)$. Then the resolvent of $\mathrm{I}$ and $\mathrm{J}$ on $x$ will be denoted as $\mathrm{I} \otimes_{x} \mathrm{~J}$.

\section{Lower Bound Backjump Conflict (LBBC):}

$$
V x^{u} U\left\|\mathcal{L},\{x+c \geq 0\} \Rightarrow_{L B B C} V\right\| \mathcal{L},\{\mathrm{b}\}, \text { where }
$$

1. $\operatorname{CCI}_{(x+c \geq 0)}$ is of the form $x+I \geq 0$,

2. $x+c \geq 0$ is inconsistent with the upper bound $u b_{x}$,

3. $\mathrm{b}$ is decision-derived from $\mathrm{CCI}_{(x+c \geq 0)} \otimes_{x} U_{x}$.

In the (LBBC) rule above, with the new decision conflicting bound $b$ we associate the conflicting collapsing inequality $\operatorname{CCI}_{(x+c \geq 0)} \otimes_{x} U_{x}$.

The rules (UBBV) and (UBBC) below are defined similarly to (LBBV) and (LBBC).

\section{Upper Bound Backjump Value (UBBV):}

$$
V x^{u} U\left\|\mathcal{L},\{-x+c \geq 0\} \Rightarrow_{U B B V} V x^{v}\right\| \mathcal{L}, \text { where }
$$

1. $\operatorname{CCI}_{(-x+c \geq 0)}$ is of the form $-x+I \geq 0$,

2. $-x+c \geq 0$ is consistent with the lower bound $l b_{x}$,

3. define $B C I_{x}^{\prime}=\left\langle\left\{\operatorname{CCI}_{(-x+c \geq 0)}\right\}, L S_{x}\right\rangle$,

4. $x^{v}$ is consistent with $u b_{x}^{\prime}$ and $l b_{x}^{\prime}$ corresponding to $B C I_{x}^{\prime}$.

In the (UBBV) rule above, with the new decision pair $x^{v}$ we associate the bounding collapsing pair $B C I_{x}^{\prime}$.

\section{Upper Bound Backjump Conflict (UBBC):}

$$
V x^{u} U\left\|\mathcal{L},\{-x+c \geq 0\} \Rightarrow_{U B B C} V\right\| \mathcal{L},\{\mathrm{b}\} \text {, where }
$$

1. $\operatorname{CCI}_{(-x+c \geq 0)}$ is of the form $-x+I \geq 0$,

2. $-x+c \geq 0$ is inconsistent with the lower bound $l b_{x}$, 
3. $\mathrm{b}$ is decision-derived from $\operatorname{CCI}_{(-x+c \geq 0)} \otimes_{x} L_{x}$.

In the (UBBC) rule above, with the new decision conflicting bound $b$ we associate the conflicting collapsing inequality $\mathrm{CCI}_{(-x+c \geq 0)} \otimes_{x} L_{x}$.

A BPA transition is a transition by one of the BPA rules above, denoted as $\Rightarrow$. A $B P A$ derivation is a sequence of BPA transitions starting from an initial state.

Let us remark on some properties of the BPA derivations. First we note that the set of inequalities $\mathcal{L}$ is never changed during the BPA derivations. Secondly, the number of inequalities at each state is linear in the number of variables and the number of input inequalities. Indeed, the only inequalities in a state are the input inequalities, inequalities in bounding collapsing pairs, which are at most double in the number of variables and at most one conflicting collapsing inequality. Thirdly, the order of variables is not fixed and can be dynamically changed during the BPA derivations. Fourthly, we can note that bounding collapsing pairs are used only during backjumping rules. Therefore in an implementation instead of computing $B C I$ 's for each decision variable, we can compute $B C I$ 's on demand during backjumping.

\section{Example}

We use a simplified notation for bounds: upper bounds of the form $-x-c \geq 0$ will be denoted as $-x \geq c$ and lower bounds of the form $x-c \geq 0$ as $x \geq c$.

Let us apply our BPA algorithm to the following set of inequalities $\mathcal{L}$.

$$
\begin{array}{r}
x_{0}-2 x_{1}-1 \geq 0 \\
x_{0}+2 x_{1}-1 \geq 0 \\
-x_{0}+x_{1} \geq 0
\end{array}
$$

We have the following possible BPA derivation.

$$
\begin{array}{lll}
\| \mathcal{L} & \Rightarrow_{D} & \\
\left\langle x_{0}^{0}\right\rangle \| \mathcal{L} & \Rightarrow_{B P}^{(6)} & \\
\left\langle x_{0}^{0}, x_{1} \geq^{p} 1 / 2\right\rangle \| \mathcal{L} & \Rightarrow_{C B}^{(5)} & \\
\left\langle x_{0}^{0}, x_{1} \geq^{p} 1 / 2\right\rangle \| \mathcal{L},\left\{x_{0} \geq 1\right\} & \Rightarrow_{L B B V} & \left(B C I^{\prime}=\left\langle\emptyset,\left\{x_{0} \geq 1\right\}\right\rangle\right) \\
\left\langle x_{0}^{1}\right\rangle \| \mathcal{L} & \Rightarrow_{B P}^{(7)} & \\
\left\langle x_{0}^{1}, x_{1} \geq^{p} 1\right\rangle \| \mathcal{L} & \Rightarrow_{C B}^{(5)} & \left(\operatorname{CCI}_{\left(-x_{0} \geq 1\right)}=\left(-x_{0} \geq 1\right)\right) \\
\left\langle x_{0}^{1}, x_{1} \geq^{p} 1\right\rangle \| \mathcal{L},\left\{-x_{0} \geq 1\right\} & \Rightarrow_{U B B C} & \left(\operatorname{CCI}_{\left(-x_{0} \geq 1\right)} \otimes_{x}\left(x_{0} \geq 1\right)=(-1 \geq 0)\right) \\
\| \mathcal{L},\{-1 \geq 0\} & \Rightarrow_{\perp \perp} &
\end{array}
$$

Let us informally explain the derivation steps. There are no bounds in $\mathcal{L}$ available for bound propagation, therefore only the Decide rule is applicable to the initial state, which adds decision bounds $x_{0} \geq^{d} 0$ and $-x_{0} \geq^{d} 0$ to the bound stack. With this decision pair we associate the empty bounding collapsing interval $\left(B C I_{x_{0}}=\langle\emptyset, \emptyset\rangle\right)$. Now, the Bound Propagation rule is applicable resulting in a propagation bound $x_{1} \geq^{p} 1 / 2$, derived from the decision bound $-x_{0} \geq^{d} 0$ and inequality (6). Next, the contradictory bound $-1 \geq 0$ is derivable by bound-resulting resolution from bounds $-x_{0} \geq^{d} 0$, $x_{1} \geq^{p} 1 / 2$ and inequality $(5)$ (transition $\Rightarrow_{C B}$ ). 
We construct a collapsing inequality for this bound as in the proof of Theorem 3.4, in this case $\mathrm{CI}_{(-1 \geq 0)}=\left(x_{0} \geq 1\right)$. Let us note that $x_{0} \geq 1$ is implied by $\mathcal{L}$ (without using any decision bounds) and all variables in $x_{0} \geq 1$ are decision variables in our bound stack. From $x_{0} \geq 1$ we decision-derive the conflicting bound $\mathrm{b}$, which in this case coincides with $x_{0} \geq 1$. We associate with $\mathrm{b}$ the conflicting collapsing inequality $\operatorname{CCI}_{\left(x_{0} \geq 1\right)}=\left(x_{0} \geq 1\right)$.

By Invariant 5 , the conflicting bound $\mathrm{b}$ contradicts to one of the decision bounds, in this case $-x_{0} \geq^{d} 0$. Next we backjump to the decision that contradicts to $\mathrm{b}$ and try to modify the decision value within the bounding collapsing interval, such that the new decision bounds would not contradict to the conflicting inequality (transition $\Rightarrow_{L B B V}$ ). This is possible by taking a decision value for $x_{0}$ satisfying the new lower collapsing bound $l b_{x_{0}}^{\prime}=\left(x_{0} \geq 1\right)$, which is decision-derived from the new collapsing interval $B C I^{\prime}=\left\langle\emptyset, \mathrm{CCI}_{\left(x_{0} \geq 1\right)}\right\rangle$. The new stack consists of the decision bounds $x_{0} \geq^{d} 1$ and $-x_{0} \geq^{d}-1$.

We apply the Bound Propagation rule adding $x_{1} \geq^{p} 1$ to the stack. Next, the contradictory bound $-1 \geq 0$ is derivable and we analyse it as above. In this case it is not possible to modify the value of the decision variable $x_{0}$ in such a way as to satisfy both the conflicting inequality and collapsing bounds on $x_{0}$. This results in application of the (UBBC) rule which resolves the conflicting inequality $\left(\mathrm{CCI}_{\left(-x_{0} \geq 1\right)}=\left(-x_{0} \geq 1\right)\right)$ with the corresponding inequality from the bounding collapsing interval $\left(L_{x_{0}}=\left(x_{0} \geq 1\right)\right.$ ). The resulting conflicting collapsing inequality is $\operatorname{CCI}_{\left(-x_{0} \geq 1\right)} \otimes_{x_{0}} L_{x_{0}}=(-1 \geq 0)$. The corresponding conflicting bound is also $(-1 \geq 0)$.

After this step, the only applicable rule is the Contradiction rule. Note that conflicting collapsing inequalities are implied by $\mathcal{L}$, and therefore at the last step we have $\mathcal{L}$ implies $-1 \geq 0$, i.e., $\mathcal{L}$ is indeed unsatisfiable.

\section{Correctness of BPA}

LEMMA 6.1 In any BPA derivation $\mathbb{S}_{0} \Rightarrow \cdots \Rightarrow \mathbb{S}_{n} \Rightarrow \cdots$, all states $\mathbb{S}_{0}, \ldots, \mathbb{S}_{n}, \ldots$ satisfy Invariants (1-7).

Proof We prove this lemma by induction on the length of the BPA derivation. Initial states satisfy the invariants since at these states the stack is empty and there are no conflicting bounds. Assume that the invariants hold for states $\mathbb{S}_{0}, \ldots, \mathbb{S}_{n-1}$. Let us show that the invariants also hold for $\mathbb{S}_{n}=S \| \mathcal{L}, \epsilon$.

Invariants (1-3) trivially follow from the definition of the BPA derivation rules.

Invariant (4) states that for any decision pair $x^{c}$ in $S$, inequalities in $B C I_{x}$ are implied by $\mathcal{L}$. This follows from the fact that inequalities in $B C I_{x}$ are either collapsing inequalities for $\mathcal{L}$, or obtained from collapsing inequalities by a sequence of resolution inferences in both cases these inequalities are implied by $\mathcal{L}$.

Let us show that Invariant (5) holds at the state $\mathbb{S}_{n}$. First, let us note the conflicting collapsing inequality $\mathrm{CCI}_{\mathrm{b}}$ is either a collapsing inequality w.r.t. $\mathcal{L}$, or obtained from collapsing inequalities by a sequence of resolution inferences, in both cases $\mathrm{CCI}_{\mathrm{b}}$ is implied by $\mathcal{L}$. Now we show that the conflicting bound $\mathrm{b}$ contradicts to a decision bound in $S$. The only non-trivial cases are when $\mathbb{S}_{n}$ is obtained by one of the BPA rules $(\mathrm{CB}),(\mathrm{LBBC})$ or $(\mathrm{UBBC})$. First we consider the $(\mathrm{CB})$ rule. If $\mathrm{b}$ is of the form 
$-1 \geq 0$ then obviously $\mathrm{b}$ is itself contradictory. Assume that $\mathrm{b}$ is of the form $l+c \geq 0$ and $\mathrm{b}$ is decision-derived from $\mathrm{CI}_{(-1 \geq 0)}$ of the form $l+I \geq 0$, where all variables in $\mathrm{CI}_{(-1 \geq 0)}$ are decision variables. Let $l+c^{\prime} \geq 0$ be the decision bound in $S$ which is used in the single bound-resulting inference deriving $-1 \geq 0$ from $\mathrm{CI}_{(-1 \geq 0)}$. It is easy to see that $\mathrm{b}$ is contradictory with $\bar{l}+c^{\prime} \geq 0$ and hence Invariant (5) is satisfied. Now we consider the (LBBC) rule. We have $\mathbb{S}_{n-1}$ is of the form $V x^{u} U \| \mathcal{L},\{x+$ $c \geq 0\}$ and $\mathbb{S}_{n}$ is of the form $V \| \mathcal{L},\{\mathrm{b}\}$. From the conditions on applicability of (LBBC) we have $x+c \geq 0$ is inconsistent with the upper bound $u b_{x}$ on $x$ and $\mathrm{b}$ is decision-derived from $\mathrm{CCI}_{(x+c \geq 0)} \otimes_{x} U_{x}$. We have $\mathrm{CCI}_{(x+c \geq 0)}$ is of the form $x+I \geq 0$, $U_{x}$ is of the form $-x+U \geq 0$. The bound $(x+c \geq 0)$ is decision-derived from $\mathrm{CCI}_{(x+c \geq 0)}$ and inconsistent with the bound $u b_{x}$, decision-derived from $U_{x}$. Therefore, $\mathrm{CCI}_{(x+c \geq 0)} \otimes_{x} U_{x}$ is inconsistent with decision bounds in $V$. Since $\mathrm{b}$ is decision-derived from CCI $(x+c \geq 0) \otimes_{x} U_{x}$, b is inconsistent with a decision bound in $V$. The case of the (UBBC) rule is similar. Therefore Invariant (5) holds at the state $\mathbb{S}_{n}$.

Invariant 6 holds by conditions in the definition of the (BP) rule.

Let us show that Invariant 7 holds at $\mathbb{S}_{n}$. We need to consider the case when $\mathbb{S}_{n}$ is obtained by one of the following BPA rules: (D), (CB), (LBBV), (UBBV), (LBBC) and (UBBC). Note that by Invariant 6 , bound propagation depth of all bounds in $S$ is less or equal to $D$. Consider the (D) rule. In this case, all inequalities in $B C I_{x}$ are collapsing inequalities obtained from bound propagation derivations of depth $\leq D$. Therefore ineqaulities in $B C I_{x}$ are in $R I_{0}$ and Invariant 6 is satisfied. Consider the (CB) rule. The bound propagation depth of the derived $(-1 \geq 0)$ is less or equal to $D+1$. Hence, $\mathrm{CCI}_{\mathrm{b}}=\mathrm{CI}_{(-1 \geq 0)}$ is in $R I_{0}$ and Invariant 6 is satisfied. In the case of the rules (LBBV) and (UBBV) one of the bounding inequalities is replaced by a conflicting collapsing inequality which is by induction assumed to be of the required resolution rank. Now we consider the case of the rule (LBBC). We have $\mathbb{S}_{n-1}$ is of the form $V x^{u} U \| \mathcal{L},\{x+c \geq 0\}$ and $\mathbb{S}_{n}$ is of the form $V \| \mathcal{L},\{\mathrm{b}\}$. Denote $S^{\prime}=V x^{u} U$. Let the conflicting bound $\mathrm{b}$ be of the form $l+c^{\prime} \geq 0$ where $\operatorname{var}(l)$ is a decision variable in $V$. The conflicting collapsing inequality associated with $\mathrm{b}$ is $\mathrm{CCI}_{(x+c \geq 0)} \otimes_{x} U_{x}$. By the induction hypothesis, $\mathrm{CCI}_{(x+c) \geq 0}$ and $U_{x}$ are in $R I_{|\operatorname{var}(\mathcal{L})|-\left|\operatorname{var}_{d}\left(S_{\leq x}^{\prime}\right)\right|}$. Hence, $\mathrm{CCI}_{(x+c \geq 0)} \otimes_{x} U_{x} \in R I_{|\operatorname{var}(\mathcal{L})|-\left|\operatorname{var}_{d}\left(S_{\leq x}^{\prime}\right)\right|+1}$. Since $V_{\leq l}$ contains at least one decision variable less than $S_{\leq x}^{\prime}$, namely $x$, we have $|\operatorname{var}(\mathcal{L})|-\left|\operatorname{var}_{d}\left(S_{\leq x}^{\prime}\right)\right|+1 \leq|\operatorname{var}(\mathcal{L})|-$ $\left|\operatorname{var}_{d}\left(V_{\leq l}\right)\right|$. Therefore, $\mathrm{CCI}_{(x+c \geq 0)} \otimes_{x} U_{x} \in R I_{|\operatorname{var}(\mathcal{L})|-\left|\operatorname{var}_{d}\left(V_{\leq l}\right)\right|}$ and Invariant 6 is satisfied. The case of the rule (UBBC) is similar.

THEOREM 6.2 (Termination) Any BPA derivation terminates.

The proof is given in Appendix C.

THEOREM 6.3 (Soundness) If a BPA derivation terminates in a contradictory state $\perp$ then the initial system of inequalities $\mathcal{L}$ is unsatisfiable.

Proof Let us note that the system of inequalities $\mathcal{L}$ does not change during BPA derivations. A BPA derivation can result in the contradictory state $\perp$ only by applying the Contradiction rule $(\perp)$. We have that the last step in such derivation is of the form $S \| \mathcal{L},\{-1 \geq 0\} \Rightarrow_{\perp} \perp$. But in this case the conflicting collapsing inequality associated with $-1 \geq 0$ is contradictory, i.e., $\mathrm{CCI}_{(-1 \geq 0)}=(-1 \geq 0)$. By Invariant 5 , $\mathrm{CCI}_{(-1 \geq 0)}$ is implied by $\mathcal{L}$, and therefore $\mathcal{L}$ is unsatisfiable. 
Before stating the Completeness theorem let us observe the following. Any BPA derivation that finishes at a state with a conflicting bound, i.e., of the form $S \| \mathcal{L},\{\mathrm{b}\}$, can be extended by applying one of the following rules: $(\perp),(\mathrm{LBBV}),(\mathrm{LBBC}),(\mathrm{UBBV})$ or (UBBC).

Theorem 6.4 (Completeness) Consider a BPA derivation $\| \mathcal{L} \Rightarrow \mathbb{S}_{1} \Rightarrow \cdots \Rightarrow \mathbb{S}_{n}$ such that $\mathbb{S}_{n}$ is a non-contradictory state of the form $S \| \mathcal{L}$ and neither the Decide rule (D) nor the Conflicting Bound rule $(\mathrm{CB})$ is applicable to $\mathbb{S}_{n}$. Then the initial system of inequalities $\mathcal{L}$ is satisfiable.

Proof Since the Decide rule is not applicable at the state $\mathbb{S}_{n}=S \| \mathcal{L}$, for any variable $x$ in $\mathcal{L}, x$ is either (i) a decision variable in $S$, or (ii) there are two implied bounds in $S$ of the form $x-c \geq 0$ and $-x+c \geq 0$, called value-implying bounds. In the latter case we call $c$ the implied value of $x$ in $S$. Let us note that by Invariant $1, S$ is consistent. Therefore each variable has a unique decision or implied value. Define an assignment $\sigma$, mapping each variable into the corresponding decision/implied value. Let us show that $\sigma$ satisfies each inequality in $\mathcal{L}$. Assume otherwise, and let $I$ be an inequality in $\mathcal{L}$ which is not satisfied by $\sigma$. It is easy to see that in this case $(-1 \geq 0)$ can be obtained by a bound-resulting resolution inference from I and the bounds in $S$, resolving all literals in I with the corresponding decision/value-implying bounds in $S$. Therefore the Conflicting Bound rule is applicable to the state $S \| \mathcal{L}$, contradicting to our assumption.

\section{Conclusions}

We presented a new method for solving systems of linear inequalities. The method incorporates DPLL-style techniques such as backjumping, lemma learning and bound propagation, which can be seen as an analogue of unit propagation in DPLL. Unlike unit propagation, bound-propagation can easily lead to non-termination if applied naively. We showed that our method is sound, complete and terminating.

\section{References}

1. M. Davis, G. Logemann, and D. W. Loveland. A machine program for theorem-proving. Commun. ACM, 5(7):394-397, 1962.

2. K. Korovin, N. Tsiskaridze, and A. Voronkov. Conflict Resolution. In I. Gent, P., editor, the 15th International Conference on Principles and Practice of Constraint Programming (CP), volume 5732 of LNCS, pages 509-523. Springer, 2009.

3. K. Korovin and A. Voronkov. Solving systems of linear inequalities by bound propagation, 2011. (full version), available at http://www.cs.man.ac.uk//korovink/my_pub/.

4. J. P. Marques-Silva and K. A. Sakallah. GRASP: a search algorithm for propositional satisfiability. IEEE Trans. Computers, 48(5):506-521, 1999.

5. K. L. McMillan, A. Kuehlmann, and M. Sagiv. Generalizing DPLL to richer logics. In the 21 st International Conference on Computer Aided Verification, volume 5643 of LNCS, pages 462-476. Springer, 2009.

6. R. Nieuwenhuis, A. Oliveras, and C. Tinelli. Solving SAT and SAT modulo theories: From an abstract Davis-Putnam-Logemann-Loveland procedure to DPLL. J. ACM, 53(6):937-977, 2006.

7. A. Schrijver. Theory of Linear and Integer Programming. John Wiley and Sons, 1998. 


\section{A Proof of Theorem 3.5}

THEOREM Let $\mathrm{b}$ be a (possibly trivial) bound derived from a context $B$ and a set $\mathcal{L}$ of linear inequalities by bound propagation. Then there exists a linear inequality I such that

\section{1. $\mathcal{L}$ implies $I$, and}

2. either (i) $I$ is $-1 \geq 0$, or (ii) there is a bound $b^{\prime}$ improving $b$ which can be derived from $B$ and I by a single bound-resulting resolution inference.

Moreover, the bound $b^{\prime}$ and inequality I can be constructed in time polynomial in the size of the derivation by bound propagation.

Proof By Theorem 3.4 one can find, in polynomial time, inequalities I and J collapsing w.r.t. b for $\mathcal{L}$ and $B$ respectively.

Let us show that I satisfies our theorem. Condition 1 is satisfied because I is collapsing for $\mathcal{L}$. Let us show that Condition 2 is also satisfied. If $I$ is $-1 \geq 0$ then Condition 2 is trivially satisfied and we are done. Assume that $I$ is different from $-1 \geq 0$. Since $I$ and $\mathrm{J}$ are collapsing w.r.t. $\mathrm{b}$ we have that $\{\mathrm{I}, \mathrm{J}\}$ implies $\mathrm{b}$. Therefore, by Theorem 3.2, there are non-negative $\alpha$ and $\beta$ such that

$$
\alpha \mathrm{I}+\beta \mathrm{J}=\mathrm{b}_{0}
$$

where $\mathrm{b}_{0}$ improves $\mathrm{b}$. Let us note that $\alpha>0$ and $\operatorname{var}(\mathrm{I}) \neq \emptyset$. Indeed, otherwise $B$ would imply $\mathrm{b}$ which contradicts to the condition that $\mathrm{b}$ is derived by bound propagation from $B$ and $\mathcal{L}$. Let us also note that $\mathrm{J}$ can be represented as a non-negative linear combination

$$
\mathrm{J}=\beta_{1} \mathrm{G}_{1}+\ldots+\beta_{m} \mathrm{G}_{m}
$$

where $\mathrm{G}_{i} \in B$ and $\beta_{i} \geq 0$ for $1 \leq i \leq m$. Let us show that there is a single boundresulting resolution inference between $I$ and bounds in $B$ deriving a bound improving $\mathrm{b}_{0}$. For this we construct a sequence $\mathrm{I}_{0}, \mathrm{~J}_{0}, \ldots, \mathrm{I}_{k}, \mathrm{~J}_{k}$ where $k=|\operatorname{var}(\mathrm{I})|-\left|\operatorname{var}\left(\mathrm{b}_{0}\right)\right|$ if $\operatorname{var}\left(\mathrm{b}_{0}\right) \subseteq \operatorname{var}(\mathrm{I})$ and $k=|\operatorname{var}(\mathrm{I})|$ otherwise. Let $\mathrm{b}_{i}$ denote $\mathrm{I}_{i}+\mathrm{J}_{i}$ for $1 \leq i \leq k$. Define $\mathrm{I}_{0}=\alpha \mathrm{I}$ and $\mathrm{J}_{0}=\beta \mathrm{J}$ (hence $\mathrm{I}_{0}+\mathrm{J}_{0}=\mathrm{b}_{0}$ ). For each $i, 0<i \leq k, \mathrm{I}_{i}$ and $\mathrm{J}_{i}$ will satisfy the following conditions.

1. $I_{i}$ can be obtained by a single resolution inference from $I_{i-1}$ and a bound in $B$. Moreover $\operatorname{var}\left(\mathrm{I}_{i}\right)=\operatorname{var}\left(\mathrm{I}_{i-1}\right) \backslash\left\{x_{i}\right\}$, where $x_{i} \in \operatorname{var}\left(\mathrm{I}_{i-1}\right) \backslash \operatorname{var}\left(\mathrm{b}_{i-1}\right)$.

2. $\mathrm{J}_{i}$ is a non-negative linear combination of bounds in $B$.

3. $\mathrm{b}_{i}$ is a bound improving $\mathrm{b}_{i-1}$.

4. $\mathrm{I}_{k}$ is a bound which is either inconsistent with $B$ or improves $\mathrm{b}_{k}$.

Let us assume that we have constructed $\mathrm{I}_{0}, \mathrm{~J}_{0}, \ldots \mathrm{I}_{i}, \mathrm{~J}_{i}$ satisfying conditions 1-3, for some $i, 1 \leq i<k$. Let us show how to construct $\mathrm{I}_{i+1}, \mathrm{~J}_{i+1}$. First note that by Condition 1, $\left|\operatorname{var}\left(\mathrm{I}_{i}\right)\right|=\left|\operatorname{var}\left(\mathrm{I}_{0}\right)\right|-i$. Let $x_{i+1}$ be a variable in $\operatorname{var}\left(\mathrm{I}_{i}\right) \backslash \operatorname{var}\left(\mathrm{b}_{i}\right)$. Then $I_{i}$ can be represented as $d l+I_{i}^{\prime} \geq 0$ where $\operatorname{var}(l)=\left\{x_{i+1}\right\}$ and $I_{i}^{\prime}$ does not contain $x_{i+1}$. From Conditions (2-3) it follows that $\mathrm{J}_{i}$ can be represented as $\beta_{1}(\bar{l}+$ $\left.c_{1}\right)+\beta_{2}\left(l+c_{2}\right)+J_{i}^{\prime} \geq 0$ where (i) $J_{i}^{\prime} \geq 0$ is a non-negative linear combination of 
bounds in $B$, (ii) $J_{i}^{\prime}$ does not contain $x_{i+1}$, (iii) $\beta_{1}>0, \beta_{2} \geq 0$, (iv) $\left(\bar{l}+c_{1} \geq 0\right) \in B$, and (v) if $\beta_{2} \neq 0$ then $\left(l+c_{2} \geq 0\right) \in B$.

Define $\mathrm{I}_{i+1}$ to be $d c_{1}+I_{i}^{\prime} \geq 0$ and $\mathrm{J}_{i+1}$ to be $J_{i}^{\prime} \geq 0$. Let us check that conditions 1-3 are satisfied by $I_{i+1}$ and $J_{i+1}$. We need to show that inequality $I_{i+1}$ can be obtained by a bound-resulting resolution inference from $I$ and bounds in $B$. Indeed, by (iv) we have $\left(\bar{l}+c_{1} \geq 0\right) \in B$. It is straightforward to check that inequality $I_{i+1}$ can be obtained by a bound-resulting resolution inference with premises $\mathrm{I}_{i} \geq 0$ and $\bar{l}+c_{1} \geq 0$. Therefore Condition 1 is satisfied. Condition 2 follows immediately from (i).

Let us show that Condition 3 is satisfied. Since $x_{i+1} \in \operatorname{var}\left(\mathrm{I}_{i}\right) \backslash \operatorname{var}\left(\mathrm{b}_{i}\right)$ and $\mathrm{b}_{i}=\mathrm{I}_{i}+\mathrm{J}_{i}$ we have $\mathrm{I}_{i}+\mathrm{J}_{i}$ does not contain $x_{i+1}$ and therefore (vi) $\beta_{1}=\beta_{2}+d$. We consider two possible cases: $\beta_{2}=0$ and $\beta_{2}>0$. Assume $\beta_{2}=0$. Then $\beta_{1}=d$ and therefore $\mathrm{b}_{i+1}=\mathrm{I}_{i+1}+\mathrm{J}_{i+1}=\mathrm{I}_{i}+\mathrm{J}_{i}=\mathrm{b}_{i}$, hence $\mathrm{b}_{i+1}$ improves $\mathrm{b}_{i}$. Now assume $\beta_{2}>0$ and therefore by $(\mathrm{v}),\left(l+c_{2} \geq 0\right) \in B$. We have

$$
\begin{aligned}
& b_{i}=I_{i}+J_{i}=d l+I_{i}^{\prime}+\beta_{1}\left(\bar{l}+c_{1}\right)+\beta_{2}\left(l+c_{2}\right)+J_{i}^{\prime} \stackrel{(\mathrm{vi})}{=} \beta_{1} c_{1}+\beta_{2} c_{2}+I_{i}^{\prime}+J_{i}^{\prime}, \\
& b_{i+1}=I_{i+1}+J_{i+1}=d c_{1}+I_{i}^{\prime}+J_{i}^{\prime}
\end{aligned}
$$

The claim follows if we show that $\beta_{1} c_{1}+\beta_{2} c_{2} \geq d c_{1}$. Indeed, by (vi) we have $\beta_{1} c_{1}+\beta_{2} c_{2}=\left(d+\beta_{2}\right) c_{1}+\beta_{2} c_{2}=\beta_{2}\left(c_{1}+c_{2}\right)+d c_{1}$. Since both $\bar{l}+c_{1} \geq 0$ and $l+c_{2} \geq 0$ are in $B$ and $B$ is consistent we have $c_{1}+c_{2} \geq 0$. Therefore, $\beta_{1} c_{1}+\beta_{2} c_{2} \geq d c_{1}$ and Condition 3 is satisfied.

Now we show that $\mathrm{I}_{k}$ satisfies Condition 4. We have $\left|\operatorname{var}\left(\mathrm{I}_{k}\right)\right|=\left|\operatorname{var}\left(\mathrm{I}_{0}\right)\right|-k \leq 1$ and therefore $\mathrm{I}_{k}$ is a bound. Since $\mathrm{I}_{k}+\mathrm{J}_{k}=\mathrm{b}_{k}$ and $\mathrm{J}_{k}$ is a non-negative linear combination of bounds from $B$, we have that $\mathrm{b}_{k}$ is implied by $\mathrm{I}_{k}$ and $B$. There are three possible cases. The first case: $I_{k}$ is $-1 \geq 0$, and in this case $I_{k}$ trivially improves $\mathrm{b}_{k}$. Assume otherwise. By the bound propagation condition $B$ does not imply $\mathrm{b}$ and hence does not imply $\mathrm{b}_{k}$. Therefore $\mathrm{I}_{k}$ is either a bound inconsistent with a bound in $B$ or $\mathrm{I}_{k}$ improves $\mathrm{b}_{k}$. In all cases Condition 4 is satisfied.

To finish the proof of the theorem let us summarise the obtained. We have $\mathcal{L}$ implies $\mathrm{I}$ and therefore implies $\mathrm{I}_{0}=\alpha \mathrm{I}$. By a sequence of resolutions with $\mathrm{I}_{0}$ and bounds in $B$ we can obtain the bound $\mathrm{I}_{k}$ and therefore $\mathrm{I}_{k}$ can be obtained by a single bound resulting resolution inference from $I_{0}$ and $B$. Since $I$ and $I_{0}$ are equivalent we can obtain a bound equivalent to $I_{k}$ by a single bound resulting resolution inference from $I$ and $B$. The bound $I_{k}$ is either (i) $-1 \geq 0$, or (ii) is inconsistent with $B$ and therefore by a single bound resulting resolution inference from $I$ and $B$ we can obtain $-1 \geq 0$, or (iii) $\mathrm{I}_{k}$ implies $\mathrm{b}_{k}$ improving $\mathrm{b}_{0}$, hence improving $\mathrm{b}$. In all cases the conclusion of the theorem holds.

\section{B Proof of Lemma 4.1}

LEMMA For any (finite) set of inequalities $\mathcal{L}$ and any non-negative integers $D$ and $k$, the set of all inequalities of resolution rank $\leq k$ is finite.

Proof We prove this lemma by induction on $k$. Let us first show that the set of all inequalities of resolution rank 0 is finite. From the proof of Theorem 3.4 it follows that the collapsing inequality extracted from a bound-propagation derivation does not depend on the value of the bounds used in that derivation. From this it follows that 
the number of collapsing inequalities extracted from bound-propagation derivations of depth $D$ is finite. Now we assume that the number of inequalities of resolution rank $\leq k-1$ is finite. Then the number of inequalities of rank $\leq k$ is bounded by the number of inequalities of rank $\leq k-1$ plus the number of all possible one step resolution inferences from inequalities of rank $\leq k-1$, which is a finite number.

\section{Proof of Theorem 6.2}

THEOREM Any BPA derivation terminates.

Proof Assume the contrary that there is an infinite BPA derivation $S_{0} \| \mathcal{L}, \epsilon_{0} \Rightarrow \ldots \Rightarrow$ $S_{n} \| \mathcal{L}, \epsilon_{n} \Rightarrow \ldots$ Let us first note that there is a constant $C$ such that for each $i \geq 0$ the length of $S_{i}$ is less than $C$. Indeed, this follows from the fact that each bound in a stack is either a decision bound or obtained from a decision bounds by bound propagation of depth $\leq D$.

Let us show that starting from some $i \geq 0$ the sub-derivation $\mathbb{S}_{i} \Rightarrow \mathbb{S}_{i+1} \ldots \Rightarrow$ $\mathbb{S}_{n} \Rightarrow \ldots$ is of the form

$$
V x^{c_{1}} U_{1}\left\|\mathcal{L}, \epsilon_{1} \Rightarrow V x^{c_{2}} U_{2}\right\| \mathcal{L}, \epsilon_{2} \Rightarrow \ldots \Rightarrow V x^{c_{n}} U_{n} \| \mathcal{L}, \epsilon_{n} \Rightarrow \ldots
$$

In other words, starting from $i$ there is no backjumping beyond the decision variable $x$. Since the bound-propagation depth is bounded by $D$, at some state $i \geq 0$ the Decide rule is applied first time, say on a variable $x$. Let us show that the sub-derivation starting from $i$ satisfies our condition. The only rules that allow to backjump beyond variable $x$ are (LBBC) and (UBBC) with the conflicting bounds of the form $x+c \geq 0,-x+c \geq$ 0 respectively. Let us consider the case of the (LBBC) rule, the case of (UBBC) is similar. We have a sub-derivation of the form $V x^{u} U\left\|\mathcal{L},\{x+c \geq 0\} \Rightarrow_{L B B C} V\right\|$ $\mathcal{L},\{\mathrm{b}\}$, where there is no decision variables in $V$. But then by Invariant 5 , b should be contradictory, i.e., of the form $-1 \geq 0$. After this step the only applicable rule is the Contradiction rule, and therefore our initial derivation would be terminating.

Consider a sub-derivation $\mathbb{D}$ of the form (10) where the fixed prefix $V$ has the greatest number of decision variables among such sub-derivations. Let us note that in $\mathbb{D}$ there are no transitions by (LBBC) nor by (UBBC) with a conflicting bound on the decision variable $x$. Let us show that starting from some state in $\mathbb{D}$ there will be no transitions by (LBBV) nor by (UBBV) with the conflicting bound on $x$. We consider only the case of the (LBBV) rule, the case of (UBBV) is similar. First we note that after each application of the (LBBV) rule with a conflicting bound on $x$, the new lower collapsing bound on $x$ strictly improves the old one. Indeed, consider an (LBBV) transition $V x^{u} U\left\|\mathcal{L},\{x+c \geq 0\} \Rightarrow_{L B B V} V x^{v}\right\| \mathcal{L}$. By Invariant $5, x+c \geq 0$ contradicts to $-x+u \geq 0$. On the other hand $-x+u \geq 0$ is consistent with $l b_{x}$. Therefore $l b_{x}^{\prime}=(x+c \geq 0)$ strictly improves $l b_{x}$. Invariant 7 implies that the resolution rank of inequalities in $B C I_{x}$ is bounded by $k=|\operatorname{var}(\mathcal{L})|-\left|\operatorname{var}_{d}\left(V_{\leq x}\right)\right|$ which is a fixed constant during the sub-derivation $\mathbb{D}$. By Lemma 4.1 there are only a finite number of different inequalities of rank $\leq k$. Therefore it is not possible to strictly improve the lower collapsing bound on $x$ infinitely many times. This shows that starting from some state in $\mathbb{D}$ there will be no transitions by (LBBV) with the conflicting bound on $x$. 
Let $\mathbb{D}^{\prime}$ be an infinite sub-derivation of $\mathbb{D}$ for which there are no transitions by (LBBV) nor by (UBBV) with the conflicting bound on $x$. Since $\mathbb{D}^{\prime}$ is infinite, the Decision rule is applied at some step. Therefore we have a transition in $\mathbb{D}^{\prime}$ of the following form $V x^{c} U\left\|\mathcal{L} \Rightarrow_{D} V x^{c} U y^{v}\right\| \mathcal{L}$. Starting from this state we have an infinite subderivation of the form: $V x^{c} U y^{v_{1}} U_{1}\left\|\mathcal{L} \Rightarrow V x^{c} U y^{v_{2}} U_{2}\right\| \mathcal{L} \Rightarrow \ldots$, where the prefix $V x^{c} U$ is fixed. But this contradicts our condition that $V$ is the fixed prefix with the maximal number of decision variables. 